\title{
IF-over-Fiber Technology Aiming at Efficient Bandwidth Utilization and Perfect Centralized Control for Next-Generation Mobile Fronthaul Links in C-RAN Architectures
}

\author{
Shota ISHIMURA ${ }^{\dagger a}$, Member, Byung-Gon KIM ${ }^{\dagger \dagger}$, Nonmember, Kazuki TANAKA ${ }^{\dagger}$, \\ Shinobu NANBA ${ }^{\dagger}$, Members, Kosuke NISHIMURA ${ }^{\dagger}$, Senior Member, Hoon KIM ${ }^{\dagger \dagger}$, \\ Yun C. CHUNG ${ }^{\dagger \dagger}$, Nonmembers, and Masatoshi SUZUKI ${ }^{\dagger}$, Fellow
}

\begin{abstract}
SUMMARY The intermediate frequency-over-fiber (IFoF) technology has attracted attention as an alternative transmission scheme to the functional split for the next-generation mobile fronthaul links due to its high spectral efficiency and perfect centralized control ability. In this paper, we discuss and clarify network architectures suited for IFoF, based on its advantages over the functional split. One of the major problems for IFoF transmission is dispersion-induced RF power fading, which limits capacity and transmission distance. We introduce our previous work, in which high-capacity and long-distance IFoF transmission was demonstrated by utilizing a parallel intensity/phase modulators (IM/PM) transmitter which can effectively avoid the fading. The IFoF technology with the proposed scheme is well suited for the long-distance mobile fronthaul links for the 5 th generation $(5 \mathrm{G})$ mobile system and beyond.

key words: centralized RAN (C-RAN), mobile fronthaul, functional split, IF-over-fiber, dispersion-induced $R F$ power fading
\end{abstract}

\section{Introduction}

Due to rapid growth and wide spread of the Internet, network traffic has been dramatically increased to date, and will be continuously growing. To accommodate such a large traffic data, optical fiber communication technologies have evolved since 1970s, employing various types of technologies, such as erbium-doped fiber amplifier (EDFA), wavelength-division multiplexing (WDM), and digital coherent detection as shown by the red line in Fig. 1 [1]. Recently, space-division multiplexing (SDM) technologies aiming to exceed the limit of single-mode fiber (SMF) have been intensively studied to increase the capacity further. These technologies mainly target core and metro networks.

On the other hand, in optical access networks which have been developed primarily for providing wired consumer access services such as fiber-to-the-home (FTTH) or fiberto-the-curb (FTTC), simple technologies are highly desired due to the strong constraint of cost; thus, intensity modulation and direct detection (IM-DD) systems and point-

\footnotetext{
Manuscript received May 15, 2017.

Manuscript revised August 14, 2017.

Manuscript publicized October 18, 2017.

$\dagger$ The authors are with KDDI Research, Inc., Fujimino-shi, 3568502 Japan.

${ }^{\dagger \dagger}$ The author are with School of Electrical Engineering, Korea Advanced Institute of Science and Technology (KAIST), 291 Daehak-ro Yuseong-gu, Daejeon, Korea 34141.

a) E-mail: sh-ishimura@kddi-research.jp

DOI: 10.1587/transcom.2017OAI0002
}

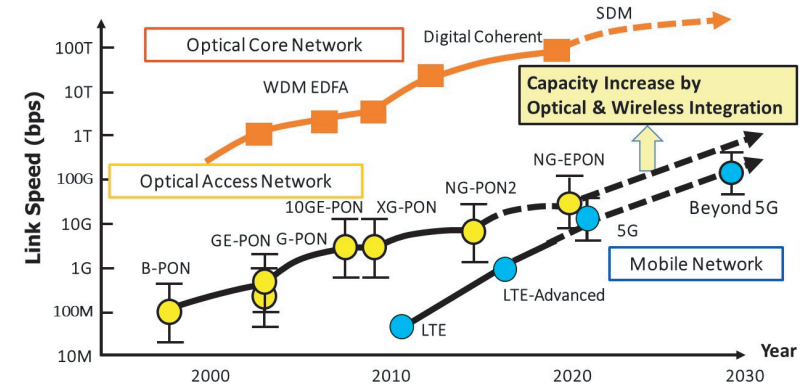

Fig. 1 Evolution of link speeds for optical core networks, optical access networks and mobile networks.

to-multipoint transmission such as passive optical network (PON) architectures have been commercially developed. At present, the maximum speed for gigabit ethernet (GE)-PON systems is about $1 \mathrm{Gbps}$, and 10GE-PON services are now about to start. However, due to the acceleration of wireless access speed, the number of subscribers of wired access services approaches a plateau of growth, whereas that of wireless access services still keeps growing constantly. Of course, to provide high-speed mobile services, optical access lines are indispensable. However, even in the 5 th generation (5G) mobile system, the maximum achievable data rate is $20 \mathrm{Gbps}$ [2], which is the comparable value to that of current FTTH services as shown in Fig. 1. If further increase in user data rate is required in the beyond-5G era, it is obvious that exiting optical access technologies will not be scalable and new technologies having high bandwidth utilization efficiency will be needed, to accommodate the huge capacity.

Unlike the conventional distributed RAN (D-RAN) architecture, baseband units (BBUs) are located at central offices (COs) to cooperatively control cells in centralized radio access network (C-RAN). Therefore, high-level coordinations can be realized through technologies such as enhanced inter-cell interference coordination (eICIC) and coordinated multi-point (CoMP) transmission and reception [3]. To connect the fronthaul link between BBU and RRH, current C-RAN mainly employs Common Public Radio Interface (CPRI), which is based on digital transmission of quantized waveforms of in-phase and quadrature (IQ) signals [4]. Such a transmission scheme is so-called "digital radio-over-fiber (D-RoF)", and it is known that the required data rate of CPRI 
is about sixteen times of the original throughput. Considering continuous growth of user data rate, apparently, CPRI is not scalable in the $5 \mathrm{G}$ mobile system and beyond, and reduction in the fronthaul capacity will be strongly required.

Several approaches to solve this issue are now being widely discussed, and one possible way is to change the functional split point between BBU and RRH. Current LTEeNodeB function consists of packet data convergence protocol (PDCP), radio-link control (RLC), medium-access control (MAC), and physical layer (PHY). According to the technical report produced by the 3rd Generation Partnership Project (3GPP) [5], eight options of the functional split are defined and studied. In terms of the required bandwidth, the cases up to the MAC-PHY split (option 6) have similar requirements. However, the intra PHY split (option 7) and the PHY-RF split (option 8: CPRI) increase the requirements significantly; thus, a split at a higher layer is desired to reduce the capacity. However, a high-layer functional split sacrifices perfect centralized control instead of the bandwidth reduction. It is a critical issue especially for future urban areas where many small cells will be deployed, and the centralized control ability will be strongly needed.

Another approach is to employ frequency-division multiplexing (FDM) with analog RoF transmission scheme, which is intermediate frequency-over-fiber (IFoF). It can keep perfect centralized control because only RF part is located in the distributed unit. In addition, IFoF has a high bandwidth utilization efficiency because radio signals are directly converted from electrical to optical domain. Therefore, the IFoF technology has attracted much attention. However, the analog-based transmission technique is much more sensitive to noise and distortion than D-RoF; in particular, dispersion-induced RF power fading is the most critical issue for them. To avoid the effect, various techniques have been proposed so far.

In this paper, we discuss network architectures suited for IFoF, based on the advantages over the functional split. Subsequently, we explain the limitation of IFoF to increase the capacity and transmission distance, namely, dispersioninduced RF power fading. Several schemes to solve it, and pros and cons of them are also explained. Finally, we introduce our previous work achieving high-capacity IFoF transmission through 40-km SMF, by utilizing a parallel intensity/phase modulators (IM/PM) transmitter with appropriate bandwidth allocations. The proposed scheme can overcome the fading with a simpler configuration than conventional parallel transmitter. Therefore, the IFoF technology with the proposed scheme is well suited for the long-distance mobile fronthaul links for $5 \mathrm{G}$ and beyond. The remainder of this paper is organized as follows. Section 2 describes concepts and network architectures for the functional split and IFoF. In Sect. 3, various types of demonstrations of IFoF are summarized in terms of throughput and transmission distance. In addition, the effect of dispersion-induced RF power fading and schemes to compensate it are discussed. Section 4 introduces our previous demonstration of the IFoF transmission with the parallel transmitter. Finally, conclusions are presented in Sect. 5.

\section{Network Architectures for Mobile Fronthaul Net- works}

In this section, we review the concept of the functional split and the network architectures. We explain difficulty of the functional split scaling up to future urban areas where many small cells will be deployed. Subsequently, we describe the concept and architectures of IFoF and its scalability to the areas.

\subsection{Architectures for the Functional Splits}

As mentioned, CPRI will not be scalable to the $5 \mathrm{G}$ era, because of its inefficient bandwidth utilization. Employing the functional split is one of the candidates to reduce the capacity. According to the technical report produced by 3GPP, eight options are defined. Before the explanation of them, the function of each protocol is briefly summarized below [6];

- PDCP compresses IP headers to reduce the number of the bits. In addition, integrity protection and ciphering are also performed here.

- RLC performs segmentation and reassembly. Furthermore, RLC has a retransmission mechanism.

- MAC is responsible for a logical mapping and hybrid ARQ retransmissions. It is also responsible for uplink and downlink scheduling.

Figures 2 and 3 show the protocol architecture for downlink and the corresponding network architectures respectively. Here, we show the architectures of option 2 and 7 as typical use cases. As shown in Fig. 3(a), a high-layer split such as option 2 does not have a good cooperative control ability because the protocol functions except for PDCP are distributed. However, it has a high bandwidth utilization efficiency, because the cases up to the MAC-PHY split (option 6) carry data with relatively small headers [7]. In contrast, as shown in Fig. 3(b), option 7 has a good cooperative control ability; however, the required bandwidth increases extremely primarily due to the IQ quantization. Therefore, it can be said that the functional split has a tradeoff relationship between centralized control ability and bandwidth utilization efficiency.

In rural areas where high-level coordination technologies are not always necessary, a high-layer split such as option 2 would be an attractive solution. In addition, the combination of the high-layer split and PON architecture will be an effective way to reduce the cost of deployment of fibers as shown in Fig. 3(c). On the other hand, in urban areas where small cells will be deployed densely, a low-layer functional split such as option 7, having good centralized control ability will be mandatory. However, as already described, due to its inefficient bandwidth utilization, even the functional split will not be able to accommodate the $5 \mathrm{G}$ signals, and of course, it is a challenging task to employ PON architecture 


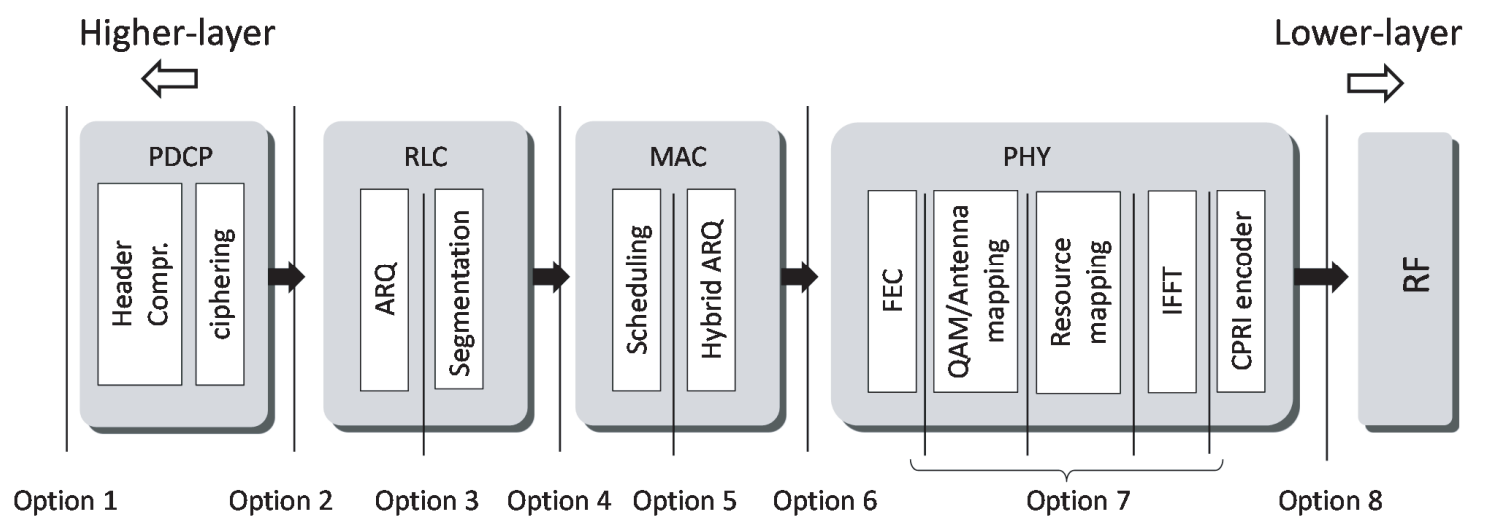

Fig. 2 Current LTE eNodeB functions for downlink, and functional splits defined in the technical report produced by $3 \mathrm{GPP}$.

(a)

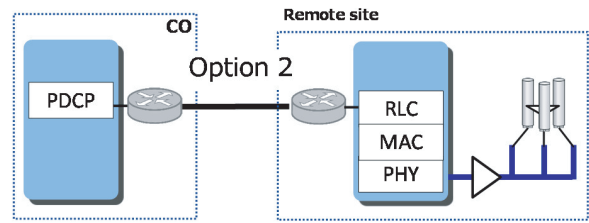

(b)

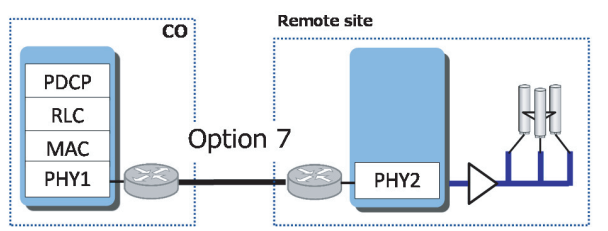

(c)

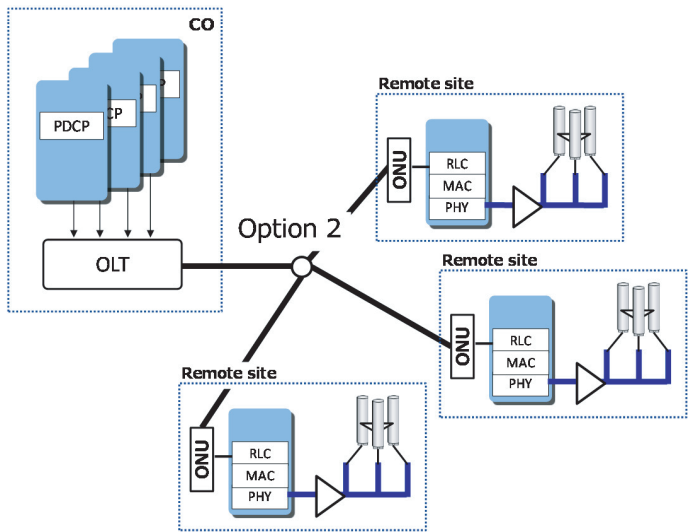

Fig. 3 Network architectures for the functional split. (a) and (b) show the cases of option 2 and 7, and (c) shows the case of option 2 with PON architecture.

with the low-layer split. In Ref. [8], only 3 CPRI signals can be accommodated in the 10G-EPON system. Thus, another new technology that can achieve both the centralized control and high bandwidth utilization efficiency will be needed.

\subsection{Architectures for IFoF}

Another technical option to reduce the fronthaul capacity is the IFoF technology. It corresponds to the option 8 (CPRI) in the functional split. However, unlike CPRI, IFoF directly delivers radio signals over fiber with digital signal processing (DSP)-assisted frequency conversion; thus, the required bandwidth is much smaller than CPRI and the functional split. For example, in the current downlink LTE system utilizing 20-MHz bandwidth (the corresponding sampling rate is $30.72 \mathrm{MHz}$ ) and $2 \times 2 \mathrm{MIMO}$, the throughput becomes about $150 \mathrm{Mbps}$; thus a high-layer function split also requires $150 \mathrm{Mbps}$. In the CPRI case, the required data rate (CPRI line bit rate option 3) is given as [11]; 2 (MIMO) $\times$ $2(\mathrm{IQ}) \times 30.72$ Mbps (sampling rate) $\times 15$ (resolution) $\times$ $16 / 15$ (CPRI overhead $) \times 10 / 8(8 \mathrm{~b} / 10 \mathrm{~b}$ encoding $)=$ $2457.6 \mathrm{Mbps}$. On the other hand, the IFoF requires the bandwidths of the radio signals themselves, namely, $2 \times 20=$ $40 \mathrm{MHz}$. If conventional binary modulation formats such as non-return-to-zero (NRZ) are employed for the D-RoF transmission, the required bandwidth of IFoF is only a sixtieth of the corresponding CPRI rate, and a third of the highlayer functional split. Therefore, by utilizing IFoF, multiple cells can be accommodated thanks to the efficient bandwidth utilization. In addition, it has a perfect centralized control ability because all protocol functions are gathered in $\mathrm{COs}$. Therefore, the IFoF technology is well suited for the use in the mobile fronthaul links especially for future urban areas where an enormous number of small cells will be deployed.

The network architectures of IFoF are shown in Fig. 4. According to Ref. [9], fronthaul links should be supported up to $20 \mathrm{~km}$. If we can increase the distance further, the number of COs can be decreased, which reduces the cost of deployments of COs; thus, here we assume that the distance between COs and remote sites are $>20 \mathrm{~km}$. Radio signals generated from BBUs are aggregated on an IF band through DSP. In the case of Fig. 4(a), after fiber transmission, the optical signal is converted to an electrical signal and retransmitted again as an optical signal at a radio aggregation unit (RAU). During this process, the signals are deaggregated, and converted to their radio frequencies by DSP. Of course, the frequency conversion can also be performed by using analog devices such as local oscillators (LOs) and mixers. As a result, each remote site does not need to have any DSP circuits and analog devices. On the other hand, unlike the optical-electrical-optical (OEO) conversion, Fig. 4(b) shows the PON architecture with IFoF. This system does not need 
(a)

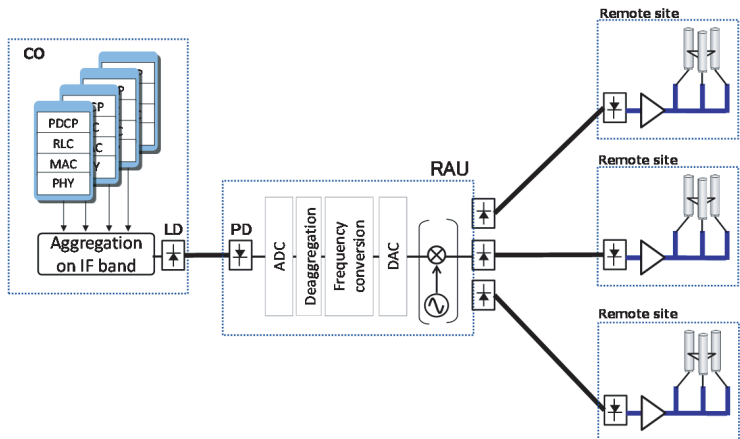

(b)

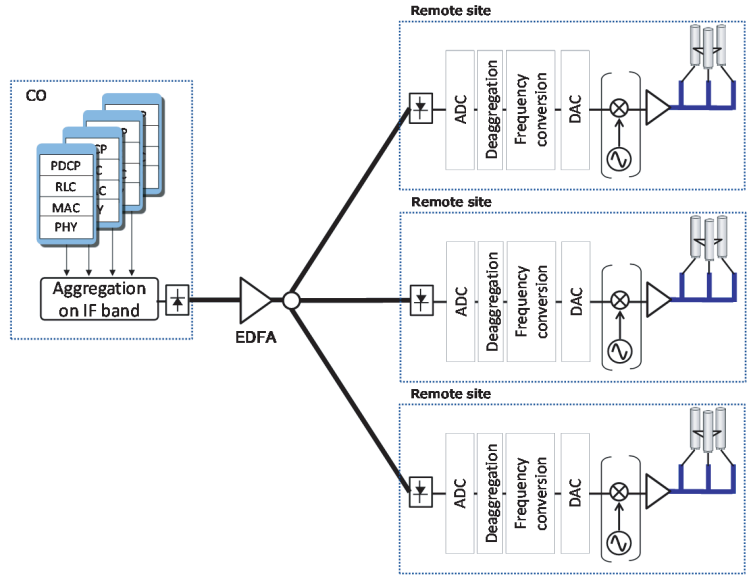

Fig. 4 Network architectures for IFoF. (a) and show the cases with RAU and PON, respectively.

any RAUs; however, the DSP circuit to deaggregate signals and frequency conversion process are needed at each remote site.

In the discussions so far, we only consider downlink transmission systems. On the other hand, an uplink transmission based on the IFoF technology is not always an easy and cost-effective solution. If we use the PON-based architecture shown in Fig. 4(b), we have three options for uplink transmissions: frequency-division multiple access (FDMA), wavelength-division multiple access (WDMA), and timedivision multiple access (TDMA). If we use an FDMA-based system, frequency conversion process to the IF band for uplink signals are also required at each remote site, which makes remote sites more complicated. In addition, the uplink signals detected by a photodiode (PD) could suffer from optical beat interference (OBI) because lights from multiple lasers arrive simultaneously [10]. On the other hand, regarding WDMA, it does not require such a frequency conversion, and OBI does not occur because other wavelength channels are removed by passing through an optical filter before a PD detection. However, a wavelength-selective laser or tunable laser is required at each remote site, which makes the system cost high. The third option is TDMA, which has been widely employed in commercial PON systems. However, applying TDMA to analog-based systems is quite challenging because analog signals from RRHs are continuous, and have to be transmitted to BBU without a pause unlike packetbased systems. On the other hand, in the architecture shown in Fig. 4(a), the frequency conversion processes for all uplink signals are performed at RAU, which keeps remote sites simple. Furthermore, we do not need to care OBI because optical signals from remote sites are detected independently.

\section{IF-over-Fiber Technologies}

In this section, we introduce various demonstrations of IFoF transmission and summarize them in terms of throughput and transmission distance. Subsequently, we explain the major problem for analog-based transmission systems, namely, dispersion-induced RF power fading, and introduce some techniques to avoid it. The pros and cons of them are also described.

\subsection{Demonstrations of IFoF Transmission}

Various papers about the IFoF technologies have been reported to date. In this subsection, we summarize them in terms of throughput and transmission distance. Their throughputs are calculated based on the 64-QAM-modulated LTE signal having $20-\mathrm{MHz}$ bandwidth (the corresponding throughput is $75 \mathrm{Mbps}$ ).

Figure 5 shows the throughputs of the works reported so far as a function of transmission distance. By using a signal having $20-\mathrm{MHz}$ bandwidth, several transmission experiments have been reported; Ref. [12] demonstrates $48 \times$ $20-\mathrm{MHz}$ signals over 5-km single-mode fiber (SMF), and Ref. [13] $20 \times 20-\mathrm{MHz}$ signals over 20-km SMF. On the other hand, Ref. [14] reports the transmission of $72 \times 20$ $\mathrm{MHz}$ signals over 30-km SMF with a frequency plan to avoid the dispersion-induced second-order distortion, and Ref. [15] $96 \times 20-\mathrm{MHz}$ signals over 20-km SMF with a dispersion compensation fiber (DCF). In Ref. [16], $24 \times 20$ $\mathrm{MHz}$ signals was successfully transmitted over $20-\mathrm{km}$ SMF with low-cost vertical-cavity surface emitting lasers (VCSEL). Reference [17] demonstrates $24 \times 20-\mathrm{MHz}$ signals over 7.2-km deployed SMF, using commercial base stations. As for IFoF transmission experiment of signals having bandwidth exceeding 20-MHz, Ref. [18] reports $6 \times 100-\mathrm{MHz}$ signals over 40-km SMF, and Ref. [19] $32 \times 200-\mathrm{MHz}$ signals over 1-km SMF.

However, a high-capacity IFoF transmission through a long-distance SMF has not been reported yet mainly because of the RF power fading. It severely degrades performance of double-sideband (DSB) signals in such conditions due to the destructive interference between the upper and lower sideband. In particular, analog-based RoF and IFoF signals are sensitive to the fading; thus, techniques which can overcome the RF power fading are necessary to increase transmission capacity and distance.

\subsection{Dispersion-Induced Power Fading Compensation Techniques}

In this subsection, we briefly explain the effect of dispersioninduced RF power fading. In addition, we introduce several 
ways to overcome it, and describe the pros and cons of them.

Here, we assume that a chirp-free Mach-Zehnder modulator (MZM) is used. The normalized frequency response of IM signal after PD detection is given as [20];

$$
P_{\mathrm{IM}}\left(\omega_{m}\right)=\cos ^{2}\left(\frac{D \lambda^{2} L}{4 \pi c} \omega_{m}^{2}\right),
$$

where $D$ is the dispersion parameter, $\lambda$ is wavelength, $L$ is fiber length, $c$ is the speed of the light, and $\omega_{m}$ is RF angular frequency. Figure 6(a) shows the frequency response as a function of fiber length and RF frequency. It is obvious that as the distance or frequency increases, the available bandwidth shrinks due to the periodic power fading. For example, if the signal is transmitted through 40-km SMF, only the frequency region up to $7 \mathrm{GHz}$ can be used.

To avoid the effect of the fading, various approaches have been proposed. They are classified mainly into two categories; one is an optical single sideband (OSSB)-based technique and the other one is a DSB-based one. Regarding the generation of the OSSB signal, it is complicated especially for wideband signals because additional DSP including the Hilbert transform [21], [22] or a narrow optical bandpass filter (OBPF) to cut one of two sidebands [23] is needed. Thus, considering actual implementation, it is difficult to employ OSSB in real scenarios.

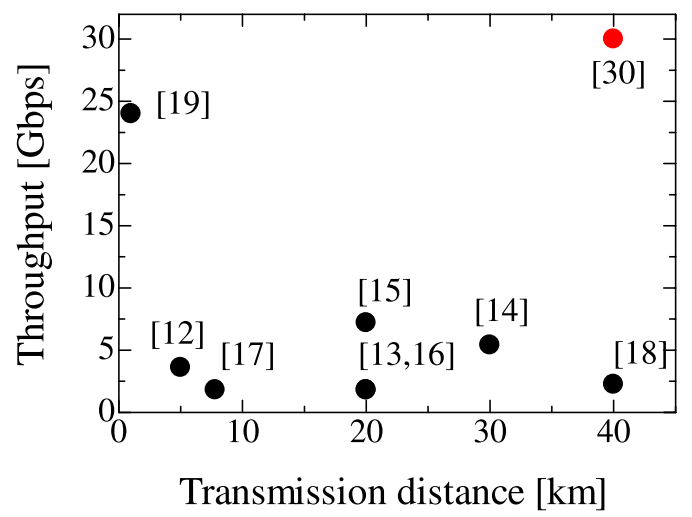

Fig.5 Throughputs of IFoF transmission demonstrated so far as a function of transmission distance.
On the other hand, regarding DSB-based techniques, schemes utilizing a dual parallel MZM (DPMZM) have been reported [24], [25]. In the schemes, by controlling DC bias voltages of DPMZM, the frequency response can also be controlled. However, they are effective only for a specific frequency range; thus, it is difficult to apply to wideband signals. Another DSB-based approach is utilizing phase modulation to intensity modulation (PM-IM) conversion through fiber transmission [26]-[28]. Reference [26] achieves the fading compensation by adjusting the state of polarization (SOP). However, this scheme can also be effective for a specific frequency like Refs. [24], [25]. In addition, the SOP manipulation has to be done manually to adjust the frequency response. On the other hand, Refs. [27], [28] introduce a transmitter consisting of parallel IM and PM. In contrast to the frequency response of IM, that of PM is given as [29];

$$
P_{\mathrm{PM}}\left(\omega_{m}\right)=\sin ^{2}\left(\frac{D \lambda^{2} L}{4 \pi c} \omega_{m}^{2}\right) .
$$

It is clear that the IM and PM responses have a complementary relationship. Figure 6(b) shows the 3-dB bandwidths of both IM and PM. As shown in the figure, PM can effectively compensate the frequency ranges which IM cannot cover alone. Utilizing this complementary relationship between the frequency responses, the transmitter can compensate the power fading to some extent in Refs. [27], [28]. However, the paths between IM and PM should be well adjusted so that the response becomes flat, which introduces high complexity in the transmitter.

\section{IF-over-Fiber Transmission with the Parallel IM/PM Transmitter}

In this section, we introduce our previous work [30]: broadband and long-distance IFoF transmission employing the parallel transmitter with appropriate bandwidth allocations to IM and PM. The proposed system overcomes the RF power fading with a simpler transmitter configuration compared to the conventional parallel transmitter. First, we explain the principle of the transmitter. Next, we show the demonstration of $20 \times 360-\mathrm{MHz}$ filtered-OFDM (f-OFDM) signals
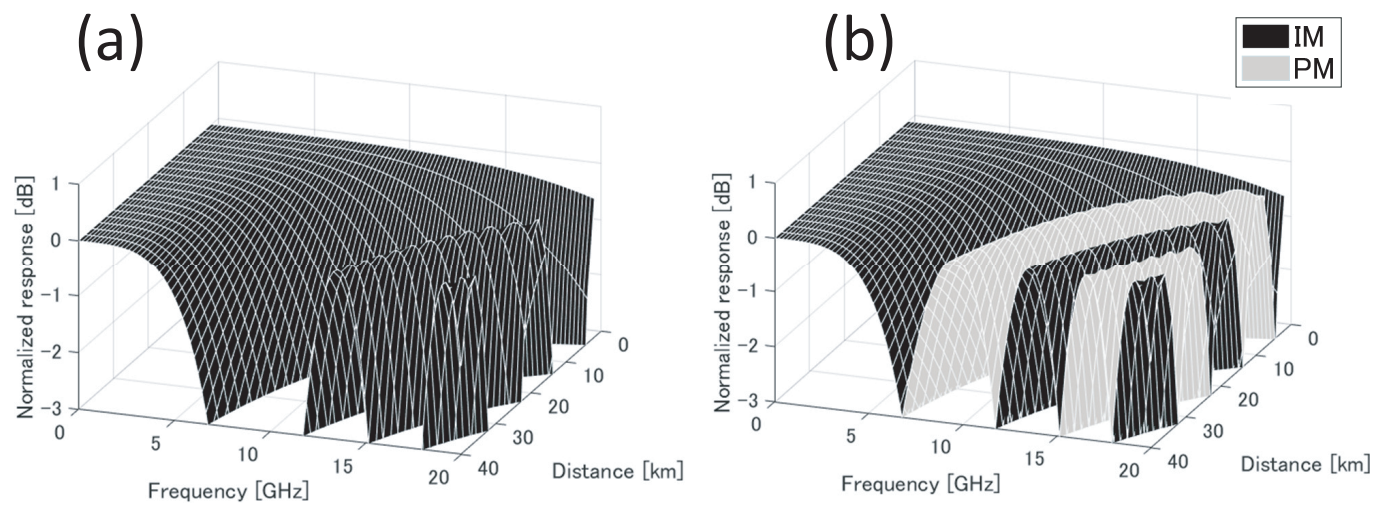

Fig. 6 3-dB responses as a function of frequency and distance. (a) shows the case of IM alone. (b) shows the case of IM and PM. 


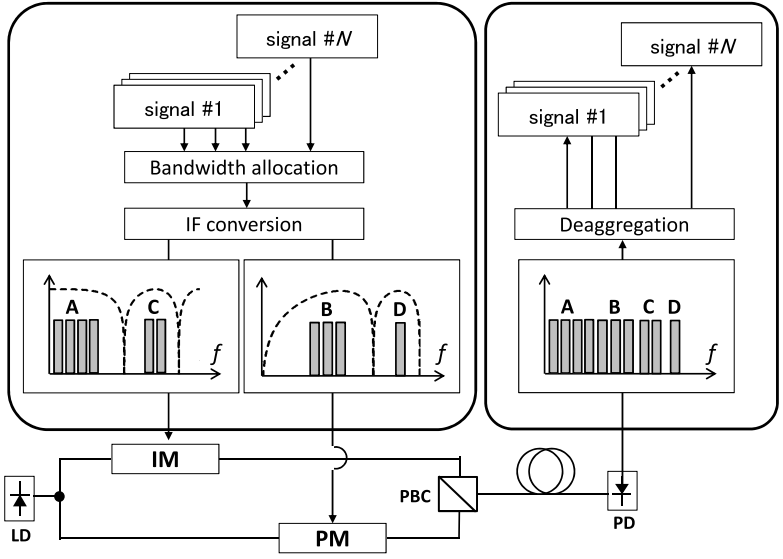

Fig. 7 Schematic of the proposed IM/PM parallel transmission system.

transmission over 40-km SMF. Finally, discussions of the result are presented.

\subsection{Principle of Parallel IM/PM Transmitter for IFoF}

Figure 7 shows a schematic of the proposed system with the parallel transmitter. After generation of baseband radio signals, they are aggregated on an IF band and assigned to either IM or PM based on measured frequency responses of IM and PM to avoid their null points. For example, in Fig. 7, the band $\mathrm{B}$ and $\mathrm{D}$ are located around the null frequencies of IM; on the other hand, the response of PM reaches its maximum values around the two bands. Thus, the band B and $\mathrm{D}$ should be assigned to PM. Note that this process can be highly flexible without dependence on the transmission distance by automatically making the decision of the bandwidth allocations. It should also be noted that in the discussions so far, we assume that IM has zero chirp. However, in practice, IMs could have non-zero chirp parameters, which causes a shrink of the available bandwidth of IM compared to the ideal chirp-free case. In the case, thus, we have to set guard bands between IM and PM. On the other hand, if we use a dual-drive MZM, by driving it in a push-pull mode with unequal amplitude signals, chirp-free signals can be generated [31], and we can reduce the guard band. After the aforementioned process, each signal is upconverted to the IF band and sent to IM or PM. Optical IM and PM signals are multiplexed on orthogonal $x$ - and $y$-polarizations by a polarization beam combiner (PBC) to avoid the interference between IM and PM. After fiber transmission, all IF channels can be detected as an IM signal because the PM signal is effectively converted to the IM. Thanks to the scheme, we can transmit broadband IF signals over long-distance fiber without being suffered from the fading. In addition, unlike Refs. [27], [28], our proposed scheme does not require any synchronization between IM and PM, which significantly reduces complexity in the transmitter.

Also for an uplink transmission, the parallel IM/PM transmitter is effective as long as RF power fading exists. For example, it can be simply applied to the connection between
$\mathrm{CO}$ and RAU in the architecture shown in Fig. 4(a). Since it is a point-to-point connection, the IM/PM transmitter for an uplink can be implemented in the same manner as the downlink. To share the information of the IF bandwidth allocations between $\mathrm{CO}$ and RAU, controlling signals have to be transmitted between them. In the parallel transmitter, the signals should be embedded around DC with IF signals and modulated by IM so that they can always avoid the effect of RF power fading regardless of transmission distance.

\subsection{Experiment and Results}

To verify the aforementioned concept, we demonstrated the transmission of $20 \times 360-\mathrm{MHz}$ f-OFDM signals over $40-\mathrm{km}$ SMF. $\mathrm{f}-\mathrm{OFDM}$ is a waveform proposed for the $5 \mathrm{G}$ mobile system to support inter-subband asynchronous transmission and mitigate an out-of-band emission [32].

Figure 8 shows the experimental setup. First, we generated 20 OFDM signals with the IFFT/FFT size of 2048. Each OFDM signal consists of 1000 subcarriers with 64QAM-modulated symbols and 200 subcarriers with pilots, and the subcarrier spacing was set to be $300 \mathrm{kHz}$. Subsequently, 7\% cyclic prefix (CP) was added, and we sent the signal to the finite impulse response (FIR) filter based on a Hamming window function with 1024 taps. As a result, we obtained $20 \mathrm{f}-\mathrm{OFDM}$ signals having $360-\mathrm{MHz}$ bandwidth. After that, they were aggregated on the IF band, and assigned to either IM or PM based on the measured frequency responses as shown in Fig. 9(a). We decided to equally assign $10 \mathrm{f}-\mathrm{OFDM}$ signals to IM and PM. For the IM band, the frequency range of the $10 \mathrm{f}-\mathrm{OFMD}$ signals was from $400 \mathrm{MHz}$ to $4 \mathrm{GHz}$. On the other hand, for the PM band, the frequency range of the other $10 \mathrm{f}$-OFMD signals was from 6.5 to $10.1 \mathrm{GHz}$. These signals were sent to arbitrary waveform generators (AWGs).

The output from a laser diode (LD) at $1550 \mathrm{~nm}$ was split into two branches by a 3-dB coupler; One for IM and the other for PM. The RF signals generated by AWGs were amplified before driving IM and PM. The spectra of the two RF signals are shown in Fig. 9(b) and (c) respectively. Two modulated optical signals having the same power were multiplexed by a PBC and transmitted with the launched power of $3 \mathrm{dBm}$. After transmission, the optical signal was detected by a PD and the output RF signal was sampled by an 80-Gsample/s digital oscilloscope. Fig. 9(d) shows the spectrum of the received RF signal. Through off-line digital signal processing (DSP), each IF signal was down-converted to the baseband and they passed through the matched filter. After the synchronization and removing the CP, FFT was performed. Finally, the EVM values were measured for all channels. Figure 10 shows them with insets of constellations at channel 1,10 , and 20 . The red diamonds and blue squares represent EVMs of signals modulated by IM and PM respectively. We also show EVMs of the electrical backto-back case as a reference. We find that although EVMs degraded by about $2 \%$ from the back-to-back case, all the EVMs satisfy $8 \%$-threshold for 64-QAM that is specified in 


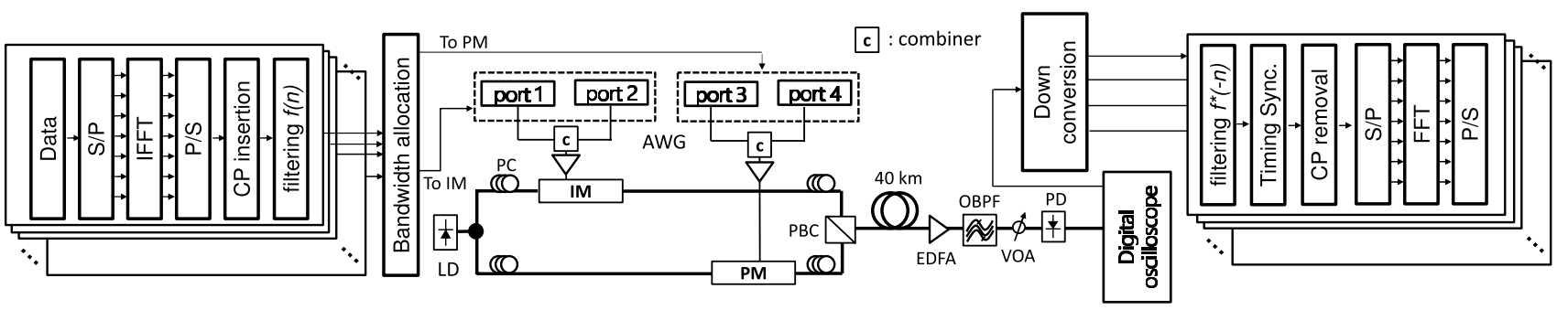

Fig. 8 Experimental setup for IFoF transmission with the parallel IM/PM transmitter.

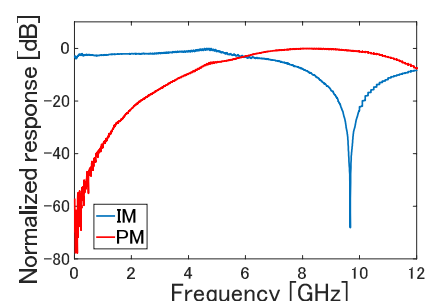

(a)

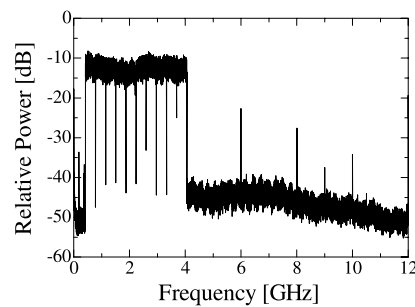

(b)

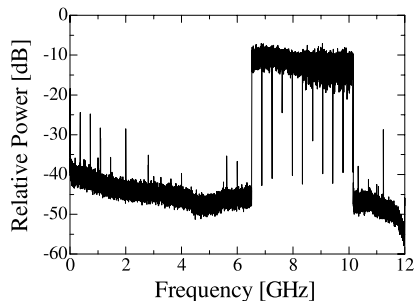

(c)

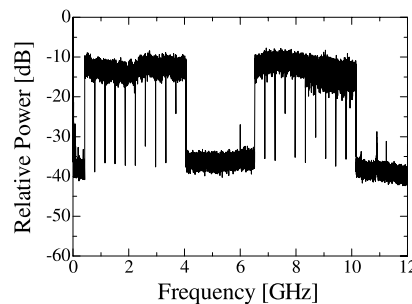

(d)

Fig. 9 Experimental results. (a) shows measured frequency responses of IM and PM after 40-km transmission. (b) and (c) show RF spectra before driving IM or PM respectively. (d) shows spectrum of received signal.

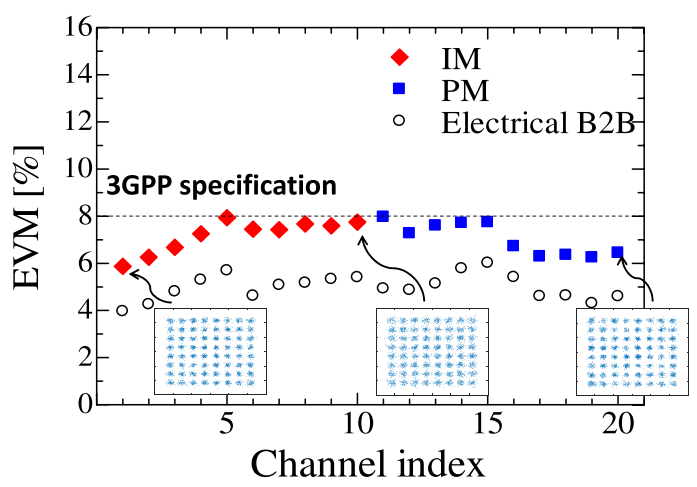

Fig. 10 Measured EVMs with the insets of constellations at channel 1, 10 and 20. Red diamonds show EVMs of IM and blue EVMs of PM. Black open circles show EVMs in electrical back-to-back case as a reference.

\section{GPP requirements [33].}

\subsection{Discussions}

In the experiment, we successfully transmitted $20 \times 360$ MHz f-OFDM signals over 40-km SMF. The corresponding throughput is $30 \mathrm{Gbps}$ as shown by a red dot in Fig. 5. This result indicates that we can achieve such a high-capacity and long-distance transmission with $10-\mathrm{GHz}$-class optical devices. If the same throughput is transmitted through 40$\mathrm{km}$ SMF by using a high-layer functional split, $30-\mathrm{GHz}$ class devices and another dispersion compensation technique are required; thus our scheme should be a cost-effective solution. Considering accommodating multiple 5G cells, even the throughput of $30 \mathrm{Gbps}$ is not enough. For example, three cells require the total throughput of $60 \mathrm{Gbps}$. In such a case, 20-GHz devices are required in the IFoF system; nevertheless it provides cost-effective solutions.

\section{Conclusions}

We have discussed the IFoF technology and clarified network architectures suited for IFoF. Since it has both high bandwidth utilization efficiency and perfect centralized control unlike the functional split, IFoF will be an effective solution for mobile fronthaul links especially in future urban area. However, the analog-based transmission has a low tolerance of noise and distortion, which limits the capacity and transmission distance. In particular, dispersion-induced RF power fading is the major issue. We described several approaches to compensate it, and pros and cons of them. Finally, we introduce our previous work, in which highcapacity and long-distance IFoF transmission was demonstrated by utilizing a parallel IM/PM transmitter which can effectively avoid the fading. The IFoF technology with the proposed scheme is scalable to the long-distance mobile fronthaul links for $5 \mathrm{G}$ and beyond.

\section{References}

[1] M. Suzuki, S. Ishimura, K. Tanaka, A. Bekkali, S. Nanba, K. Nishimura, B.G. Kim, H. Kim, and Y.C. Chung, "Optical and wireless integrated technologies for future mobile networks," International Conference on Transparent Optical Networks (ICTON2017), We.A2.2, 2017

[2] ITU-R M.2083-0, "IMT Vision - Framework and overall objectives of the future development of IMT for 2020 and beyond," 2015.

[3] K. Tanaka and A. Agata, "Next-generation optical access networks for C-RAN," Optical Fiber Communication Conference (OFC 2015), Tu2E.1, 2015.

[4] CPRI specification v7.0, 2015.

[5] 3GPP TR 38.801 v2.0.0 Release 14, 2017. 
[6] E. Dahlman, S. Parkvall, and J. Skold, 4G: LTE/LTE-Advanced for Mobile Broadband, Academic Press, 2011.

[7] L. Valcarenghi, K. Kondepu, F. Giannone, and P. Castoldi, "Requirements for 5G fronthaul," International Conference on Transparent Optical Networks (ICTON2016), We.C2.1, 2016.

[8] N. Shibata, T. Tashiro, S. Kuwano, N. Yuki, J. Terada, and A. Otaka, "Mobile front-haul employing ethernet-based TDM-PON system for small cells," Optical Fiber Communication Conference (OFC 2015), M2J.1, 2015.

[9] China Mobile Research Institute, Alcatel-Lucent, Nokia Networks, ZTE Corporation, Broadcom Corporation and Intel China Research Center, "White paper of next generation fronthaul interface," Online, 2015.

[10] S.L. Woodward, X. Lu, T.E. Darcie, and G.E. Bodeep, "Reduction of optical-beat interference in subcarrier networks," IEEE Photon. Technol. Lett., vol.8, no.5, pp.694-696, 1996.

[11] Small Cell Forum, "Small cell virtualization functional splits and use cases," Release 6.0, 159.06.02, 2016

[12] X. Liu, H. Zeng, N. Chand, and F. Effenberger, "Experimental demonstration of high-throughput low-latency mobile fronthaul supporting 48 20-MHz LTE signals with 59-Gb/s CPRI-Equivalent rate and 2-ms processing latency," European Conference on Optical Communication (ECOC2015), We.4.4.3, 2015.

[13] F. Effenberger and X. Liu, "Power-efficient method for IM-DD optical transmission of multiple OFDM signals," Opt. Express., vol.23, no.10, pp.13571-13579, 2015.

[14] C. Han, M. Sung, S.H. Cho, H.S. Chung, S.M. Kim, and J.H. Lee, "Performance improvement of multi-IFoF-based mobile fronthaul using dispersion-induced distortion mitigation with IF optimization," J. Lightwave Technol., vol.34, no.20, pp.4772-4778, 2016.

[15] M. Sung, C. Han, S.H. Cho, H.S. Chung, S.M. Kim, and J.H. Lee, "Bandwidth efficient transmission of 96 LTE-A signals with $118-\mathrm{Gb} / \mathrm{s}$ CPRI-equivalent rate using 2-GHz frequency span and intermixing mitigation," International Conference on Information and Communication Technology Convergence (ICTC2016), pp.775-777, 2016

[16] B.G. Kim, S.R. Bae, H. Kim, and Y.C. Chung, "Mobile fronthaul optical link for LTE-A system using directly-modulated 1.5$\mu \mathrm{m}$ VCSEL," OptoElectronics and Communications Conference (OECC2016), TuA2-3, 2016.

[17] B.G. Kim, K. Tanaka, T. Kobayashi, A. Bekkali, K. Nishimura, H. Kim, M. Suzuki, and Y.C. Chung, "Transmission experiment of LTE signals by IF-over-Fiber using commercial base station and deployed optical fibers," European Conference on Optical Communication (ECOC2016), W.4.P1.SC7.75, 2016.

[18] X. Liu, F. Effenberger, N. Chand, L. Zhou, and H. Lin, "Efficient mobile fronthaul transmission of multiple LTE-A signals with 36.86$\mathrm{Gb} / \mathrm{s}$ CPRI-equivalent data rate using a directly-modulated laser and fiber dispersion mitigation," Asia Communications and Photonics Conference (ACP 2014), AF4B.5, 2014.

[19] X. Liu, H. Zeng, N. Chand, and F. Effenberger, "Efficient mobile fronthaul via DSP-based channel aggregation," J. Lightwave Technol., vol.34, no.6, pp.1556-1564, 2016.

[20] G.J. Meslener, "Chromatic dispersion induced distortion of modulated monochromatic light employing direct detection," IEEE J. Quantum Electron., vol.20, no.10, pp.1208-1216, 1984.

[21] D.F. Hewitt, "Orthogonal frequency division multiplexing using baseband optical single sideband for simpler adaptive dispersion compensation," Optical Fiber Communication Conference (OFC 2007), OME7, 2007.

[22] M. Schuster, S. Randel, C.A. Bunge, S.C. J. Lee, F. Breyer, B. Spinnler, and K. Petermann, "Spectrally efficient compatible singlesideband modulation for OFDM transmission with direct detection," IEEE Photon. Technol. Lett., vol.20, no.9, pp.670-672, 2008.

[23] J. Park, W.V. Sorin, and K.Y. Lau, "Elimination of the fibre chromatic dispersion penalty on $1550 \mathrm{~nm}$ millimeter-wave optical transmission,” Electron. Lett., vol.33, no.6, pp.512-513, 1997.

[24] S. Li, X. Zheng, H. Zhang, and B. Zhou, "Compensation of dispersion-induced power fading for highly linear radio-over-fiber link using carrier phase-shifted double sideband modulation," Opt. Lett., vol.36, no.4, pp.546-548, 2011.

[25] Y. Gao, A. Wen, Y. Chen, S. Xiang, H. Zhang, and L. Shang, "An analog photonic link with compensation of dispersion-induced power fading," IEEE Photon. Technol. Lett., vol.27, no.12, pp.1301-1304, 2015

[26] Y. Gao, A. Wen, L. Liu, S. Tian, S. Xiang, and Y. Wang, "Compensation of the dispersion-induced power fading in an analog photonic link based on PM-IM conversion in a Sagnac loop," J. Lightwave Technol., vol.33, no.13, pp.2899-2904, 2015.

[27] V.J. Urick and F. Bucholtz, "Modulation diversity for chromatic dispersion compensation in analog photonic links," NRL Memorandum Report, NRL/MR/5650-06-8977, 2006.

[28] Y. Cui, K. Xu, J. Dai, X. Sun, Y. Dai, Y. Ji, and J. Lin, "Overcoming chromatic-dispersion-induced power fading in ROF links employing parallel modulators," IEEE Photon. Technol. Lett., vol.24, no.14, pp.1173-1175, 2012

[29] H. Chi, X. Zou, and J. Yao, "Analytical models for phase-modulationbased microwave photonic systems with phase modulation to intensity modulation conversion using a dispersive device," J. Lightwave Technol., vol.27, no.5, pp.511-521, 2009.

[30] S. Ishimura, B.G. Kim, K. Tanaka, K. Nishimura, H. Kim, Y.C Chung, and M. Suzuki, "Broadband IF-over-fiber transmission with parallel IM/PM transmitter overcoming dispersion-induced RF power fading for high-capacity mobile fronthaul links," IEEE Photonics J., vol.10, no.1, pp.1-9, 2018.

[31] H. Kim and A.H. Gnauck, "Chirp characteristics of dual-drive MachZehnder modulator with a finite DC extinction ratio," IEEE Photon. Technol. Lett., vol.14, no.3, pp.298-300, 2002.

[32] X. Zhang, M. Jia, L. Chen, J. Ma, and J. Qiu, "Filtered-OFDM enabler for flexible waveform in the 5th generation cellular networks," Global Communications Conference (GLOBECOM2015), pp.1-6, 2015

[33] 3GPP TS 36.104 v12.5.0 Release 12, 2014.

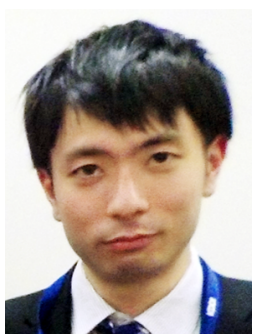

Shota Ishimura received his B.E. and M.E. degrees in Electrical Engineering from Tokyo University of Agriculture and Technology, and the University of Tokyo in 2013 and 2015, respectively. He joined KDDI R\&D Laboratories, Inc. (currently KDDI Research, Inc.), Saitama, Japan, in 2016, and has been engaged in research on optical access networks. He is a member of the Institute of Electronics, Information and Communication Engineers (IEICE).

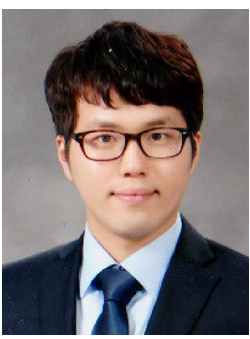

Byung Gon Kim received his B.S. and M.S. degrees Department in Electrical Engineering from Hongik university and KAIST in 2014 and 2016 respectively. He is currently pursuing the Ph.D. degree in Electrical Engineering from KAIST. His research interest is analog radioover-fiber technology. 


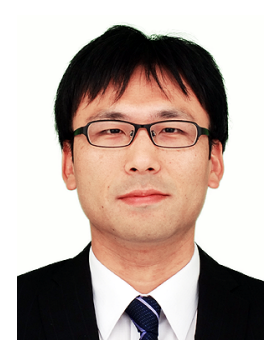

Kazuki Tanaka received the B.E. and M.E. degrees in applied physics from Waseda University in 2004 and 2006, respectively. He joined KDDI R\&D Laboratories, Inc. (currently KDDI Research, Inc.), Saitama, Japan, in 2006, and has been engaged in research on optical access networks and microwave photonics technologies. $\mathrm{He}$ is a member of the Institute of Electronics, Information and Communication Engineers (IEICE).

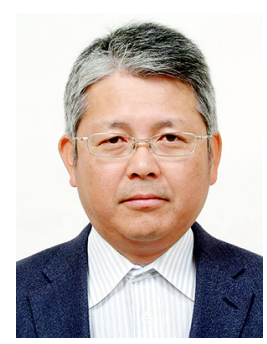

Shinobu Nanba received the B.E., M.E., and Ph.D. degrees, in information science and electrical engineering from Kyushu University, Fukuoka, Japan, in 1994, 1996, and 2006, respectively. He joined Kokusai Denshin Denwa Co., Ltd. (now KDDI Corp.) in 1996. He is currently a R\&D manager of Optical Access Network Laboratory in KDDI Research, Inc. His research interests include optical and wireless access networks and both fronthaul and backhaul design for future networks.

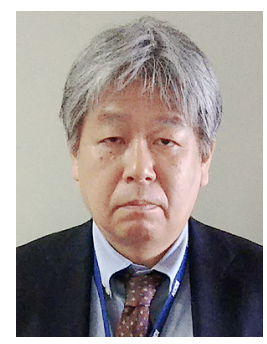

Kosuke Nishimura received B.E., M.S. and Ph.D. degrees in electrical engineering from Tokyo Institute of Technology, Tokyo, Japan in 1986, 1988, and 2008, respectively. Since 1988 , has been with KDDI R\&D Laboratories, Saitama, Japan where he has studied visible light emitting materials including II-VI semiconductors and porous silicon, and all-optical functional devices for future photonic network. He also engaged in the research and development of new applications utilizing novel user-interface devices such as electronic paper display. Currently, he is engaged in the research of optical network technologies. He is a member of Society for Information Display (SID), Japan Society of Applied Physics (JSAP), and IEICE.

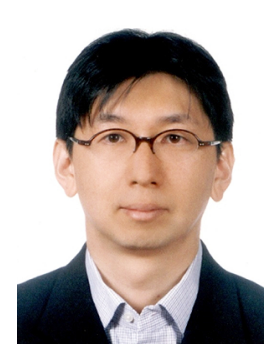

Hoon Kim is an Associate Professor of the School of Electrical Engineering at Korea Advanced Institute of Science and Technology (KAIST). Prior to joining KAIST in 2014, he was with Bell Labs, Lucent Technologies (2000-2001), Samsung Electronics, Korea (2001-2007), and National University of Singapore (2007-2014). He currently serves as Associate Editors of IEEE Photonics Technology Letters and Optics Express. His research interests include high capacity fiber-optic communication systems, broadband optical access networks, mobile fronthaul/backhaul networks, and lightwave subsystems.

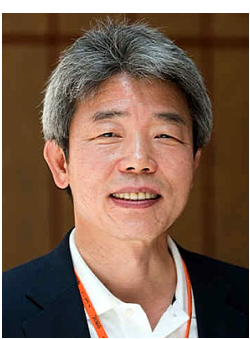

Yun C. Chung is professor of electrical engineering at the Korea Advanced Institute of Science and Technology (KAIST), which he joined in 1994. From 1987 to 1994, he was with the Lightwave Systems Research Department at AT\&T Bell Laboratories. From 1985 to 1987, he was with Los Alamos National Laboratory under AWU-DOE Graduate Fellowship Program. His current research activities include high-capacity WDM systems and networks, optical performance monitoring techniques, WDM passive optical networks, and fiber-optic networks for wireless communications, etc. He has published over 500 journal and conference papers in these areas and holds over 90 patents. He has been General Co-Chair of OFC, OECC, and APOC, and served as the President of the Optical Society of Korea. Prof. Chung is a Fellow of IEEE, OSA, Korean Academy of Science and Technology, and The National Academy of Engineering of Korea.

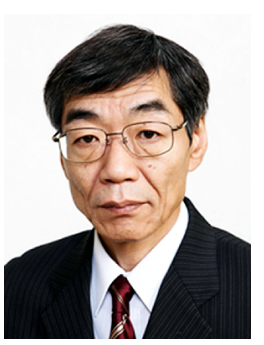

Masatoshi Suzuki received his Ph.D. from Hokkaido University. He joined KDD Research Laboratories (currently KDDI Research), Tokyo, Japan in 1984. Since then, he has been engaged in research on high-speed optical devices, optical transmission systems and optical networks. Currently, he is the Principal Research Engineer of KDDI Research, Inc., and an R\&D Fellow of KDDI Corporation. Dr. Suzuki is the Fellows of IEEE, OSA and IEICE. He is a recipient of Distinguished Paper Award of IEICE in 1996, Achievement award from IEICE in 2004, Minister Award on Science and Technology from MEXT (Ministry of Education, Culture, Sports, Science and Technology of Japan) in 2006, Minister Award of Advanced Technology from METI (Ministry of Economy, Trade and Industry of Japan) in 2006, Sakurai Memorial Award from OITDA in 2009, Hisoka Maejima Award from Tsushinbunka Association in 2011, and the Medal of Honor with Purple Ribbon from Japan's Cabinet Office in 2017. 